\title{
Activity and safety of NGR-hTNF, a selective vascular-targeting agent, in previously treated patients with advanced hepatocellular carcinoma
}

\section{A Santoro ${ }^{1,6}$, T Pressiani ${ }^{1,6}$, G Citterio², G Rossoni ${ }^{2}$, G Donadoni ${ }^{2}$, F Pozzi ${ }^{2}$, L Rimassa', N Personeni ${ }^{1}$, S Bozzarelli', G Rossoni ${ }^{3}$, S Colombi ${ }^{3}$, FG De Braud ${ }^{4}$, F Caligaris-Cappio ${ }^{2,5}$, A Lambiase and C Bordignon*,3,5 $^{3}$ \\ 'Department of Oncology, Istituto Clinico Humanitas, Rozzano, Italy; ' Department of Oncology, Istituto Scientifico San Raffaele, Milan, Italy; ${ }^{3}$ MolMed, Milan, Italy; ${ }^{4}$ Division of Clinical Pharmacology and New Drugs, European Institute of Oncology, Milan, Italy; ${ }^{5}$ Università Vita-Salute San Raffaele, Via Olgettina, Milan 20132, Italy}

BACKGROUND: Hepatocellular carcinoma (HCC) is a highly vascularised and poor-prognosis tumour. NGR-hTNF is a vascular-targeting agent consisting of human tumour necrosis factor-alpha fused to the tumour-homing peptide NGR, which is able to selectively bind an aminopeptidase $\mathrm{N}$ overexpressed on tumour blood vessels.

METHODS: Twenty-seven patients with advanced-stage disease resistant to either locoregional (59\%; range, I-3), systemic treatments (52\%; range, I-3) or both (33\%) received NGR-hTNF $0.8 \mu \mathrm{g} \mathrm{m}^{-2}$ once every 3 weeks. The primary aim of the study was progression-free survival (PFS).

RESULTS: No grade 3-4 treatment-related toxicities were noted. Common toxicity included mild-to-moderate, short-lived chills (63\%). Median PFS was 2.3 months (95\% Cl: I.7-2.9). A complete response ongoing after 20 months was observed in a sorafenibrefractory patient and a partial response in a Child-Pugh class-B patient, yielding a response rate of $7 \%$. Six patients (22\%) experienced stable disease. The disease control rate (DCR) was 30\% and was maintained for a median PFS time of 4.3 months. Median survival was 8.9 months (95\% Cl: $7.5-10.2)$. In a subset of 12 sorafenib-resistant patients, the response rate was $8 \%$ and the median survival was 9.5 months.

CONCLUSION: NGR-hTNF was well tolerated and showed single-agent activity in HCC. Further investigation in HCC is of interest. British Journal of Cancer (2010) I 03, 837-844. doi:I0.I038/sj.bjc.6605858 www.bjcancer.com

Published online 17 August 2010

(c) 2010 Cancer Research UK

Keywords: NGR-hTNF; vascular-targeting agent; hepatocellular carcinoma

Hepatocellular carcinoma (HCC) ranks as the third most common cause of cancer-related death worldwide. A majority of HCC patients present with advanced-stage disease (class $\mathrm{C}$ ) according to Barcelona Clinic Liver Cancer (BCLC) classification and are ineligible for potential radical therapy or transarterial chemoembolisation (Llovet et al, 2008a). Recently, sorafenib, an oral multikinase inhibitor against vascular endothelial growth factor receptor-2 and Ras/Raf kinase, was found to delay tumour progression and improve survival in two large randomised trials in patients with advanced HCC, leading to its use as first-line treatment in this setting (Llovet et al, 2008b; Cheng et al, 2009). Although earlier studies suggested the relevance of the Ras-Raf pathway in primary liver tumours, more recently reported data suggested that a major mode of action of sorafenib in the treatment of HCC is based on its antiangiogenic potential. In fact, hypervascularity is a prominent clinical feature of HCC and a high microvessel density has been associated with poor prognosis,

\footnotetext{
*Correspondence: Dr C Bordignon; E-mail: claudio.bordignon@hsr.it

${ }^{6}$ These two authors contributed equally to this work.

Revised 7 July 2010; accepted 20 July 20 I0; published online 17 August 2010
}

thus rendering a vascular-targeting approach appealing for HCC treatment (Greten et al, 2009).

Since its discovery (Carswell et al, 1975), tumour necrosis factor-alpha $(\mathrm{TNF} \alpha)$ has shown a powerful antivascular activity, which is mainly mediated by apoptosis of tumour-associated endothelial cells. In order to reproduce these effects, progressively increasing doses of $\mathrm{TNF} \alpha$, given with a variety of schedules, were tested in the early-stage clinical development. However, these initial dose-finding studies resulted in severe toxicity when TNF $\alpha$ was administered systemically, and the maximum-tolerated dose (MTD) being 10 times lower than the estimated effective dose. More recently, isolated limb or liver perfusion were tested as a way by which they reach high $\mathrm{TNF} \alpha$ concentrations in localised areas. Consistently, locoregional delivery of high doses of TNF $\alpha$ and chemotherapy induced elevated response rates in patients with advanced tumours of the extremities as well as regression of primary and metastatic liver tumours (Lejeune et al, 2006).

To exploit a ligand-directed, vascular-targeting approach, NGRhTNF was prepared by fusing TNF $\alpha$ with the tumour-homing peptide asparagine-glycine-arginine (NGR), which is able to selectively bind an aminopeptidase $\mathrm{N}$ (CD13) isoform overexpressed by endothelial cells of many tumours, including HCC (Arap et al, 1998; Curnis et al, 2000; Corti and Ponzoni, 2004). 
CD13 is involved in chemokine processing, tumour invasion, and angiogenesis, and it was shown to be crucial for pathological development of newly formed blood vessels from pre-existing blood vessels in a CD13-null mice model (Rangel et al, 2007).

Preclinically, murine NGR-TNF induced antitumour effects 10 -fold higher than the untargeted $\mathrm{TNF} \alpha$, without evidence of increased toxicity. It is worth noting that this conjugate showed antitumour activity even when used at doses as low as $0.005 \mu \mathrm{g} \mathrm{kg}^{-1}$ (Curnis et al, 2002a), which is equivalent in humans to the phase I starting dose of $0.2 \mu \mathrm{g} \mathrm{m}^{-2}$.

The MTD of NGR-hTNF when administered once every 3 weeks was established at $45 \mu \mathrm{g} \mathrm{m}^{-2}$ in a phase I study evaluating a dose interval from 0.2 to $60 \mu \mathrm{g} \mathrm{m}^{-2}$ (van Laarhoven et al, 2010). Firstcycle dose-limiting toxicities included transient dyspnoea and acute infusion reaction. An additional study, further exploring the low-dose range from 0.2 to $1.6 \mu \mathrm{g} \mathrm{m}^{-2}$, select $0.8 \mu \mathrm{g} \mathrm{m}^{-2}$ as the optimal biological dose based on more pronounced dynamic imaging changes, lack of TNF receptors shedding, safety, and preliminary antitumour activity (Gregorc et al, 2010). In this low-dose study, tumour blood flow and perfusion as assessed by dynamic contrast-enhanced magnetic resonance imaging (DCE-MRI) were significantly decreased. Of note, these reductions correlated with radiologically assessed disease stabilisation, thus suggesting that changes in tumour vascularity might be a determinant of disease control associated with NGR-hTNF administration.

In light of both the angiogenic profile of HCC and the antivascular effect of NGR-hTNF, the current phase II was designed to assess activity and safety of NGR-hTNF given at $0.8 \mu \mathrm{g} \mathrm{m}^{-2}$ in previously treated patients with advanced HCC.

\section{PATIENTS AND METHODS}

\section{Eligibility criteria}

Patients were required to have histologically confirmed, advanced or metastatic HCC. Other eligibility requirements included surgically unresectable disease; age 18 years or greater; Eastern Cooperative Oncology Group performance status of $0-1$; no more than one previous systemic therapeutic regimen; documented progressive disease at study entry; and adequate organ function, including hepatic (total bilirubin $<2$-fold of the upper limit of normal (ULN), transaminases $<3$-fold of ULN, and Child-Pugh class-A or -B liver dysfunction with no refractory ascites or encephalopathy), renal (serum creatinine $<1.5$-fold of ULN), and haematological (absolute neutrophil count $>1.5 \times 10^{9} 1^{-1}$, platelets $\left.>100 \times 10^{9} 1^{-1}\right)$. Exclusion criteria included clinically significant cardiac disease and uncontrolled hypertension. All patients gave written informed consent and the study was approved by the Ethics Committees of the participating institutions.

\section{Study design and dosing}

This was a multicentre, single-agent phase II trial evaluating two treatment schedules of NGR-hTNF in two sequential cohorts. The primary objective of this study was to evaluate the antitumour activity of NGR-hTNF in terms of progression-free survival (PFS), defined as the time from baseline to the first evidence of radiological or clinical progression, or death owing to any cause, whichever occurred first. The date of last radiological assessment carried out within 14 days before the first administration was considered as baseline. Tumour restaging was conducted every 6 weeks and measurable target lesions were assessed by the standard WHO criteria. Secondary study aims were: disease control rate (DCR), which included the proportion of patients experiencing complete response, partial response, or stable disease; overall survival (OS), defined as the time from baseline to death from any cause, or last contact; and safety, with adverse events (AEs) graded according to the Common Terminology Criteria for Adverse Events, version 3.0.

NGR-hTNF was given intravenously as an 1-h infusion at the dose of $0.8 \mu \mathrm{g} \mathrm{m}^{-2}$ every 3 weeks (triweekly schedule). On completion of study accrual, based on the relatively short drug half-life and the infusion-related toxicity profile of NGR-hTNF, a new cohort of 12 patients was enrolled to explore the safety of a more dense dosing schedule of NGR-hTNF given at same dose of $0.8 \mu \mathrm{g} \mathrm{m}^{-2}$ on a weekly basis. This weekly schedule was considered safe if one or fewer of six patients after a first step and two or fewer of 12 patients had grade 4 haematological or grade 3-4 non-haematological toxicity.

The duration of the treatment was related to the clinical outcome. In case of stable disease or objective response, the study treatment was continued until progressive disease, unacceptable toxicity or patient's refusal. No formal dose reduction for NGR-hTNF was planned. In the presence of grade 3-4 toxicity, the treatment could be either delayed or discontinued at the discretion of the investigator, depending on the toxicity type.

\section{Statistical analysis}

The primary end point of the study was the 12-week progressionfree rate. The sample size was based on the Simon two-stage design, assuming $\alpha=10 \%, \beta=10 \%, P_{0}=35 \%$, and $P_{1}=60 \%$. Given these criteria, 16 and a total of 27 patients were to be enrolled after the first and second stage, respectively, and study treatment could be considered worthy of additional testing if seven (44\%) and 13 patients (48\%) were progression free at 12 weeks after the first and second stage, respectively. All efficacy analyses were performed by using an intent-to-treat principle. Descriptive statistics were used to characterise patients at baseline. Time-toevent end points were estimated by Kaplan-Meier methodology. An exploratory, univariate Cox regression model was used to examine the impact of defined baseline characteristics on survival time. The variables included: age (as continuous variable), gender, PS (0 vs 1), Child-Pugh class (A vs B), BCLC stage (B vs C), Cancer of the Liver Italian Program (CLIP) score (0-3 vs 4), and serum alpha-fetoprotein (lower $v s$ equal or higher than the median value). All analyses were performed with SPSS 13.0 (SPSS Inc., Chicago, IL, USA). ClinicalTrials.gov registration is NCT00484211.

\section{RESULTS}

\section{Patients}

Twenty-seven patients were enrolled in three centres between February 2007 and June 2008 in the triweekly cohort. Median patient age was 67 years (range, 34-79). From June 2008 to February 2009, an additional 13 patients with a median age of 68 years (range, 54-78) were enrolled in a subsequent cohort treated with NGR-hTNF given at $0.8 \mu \mathrm{g} \mathrm{m}^{-2}$ on a weekly basis. A subset of 12 patients, including five and seven patients enrolled in the triweekly and weekly cohorts, respectively, were previously treated with sorafenib. Patient characteristics and previous treatments for the triweekly, weekly, and sorafenib-resistant cohorts are listed in Table 1.

\section{Safety}

All patients received at least one dose of NGR-hTNF given every 3 weeks and resulted assessable for toxicity. A total of 99 cycles (mean, 3.7; median, 2; range, 1-27) were delivered. Twenty-six patients $(96 \%)$ discontinued because of clinical ( $n=2$; Child-Pugh progression and impaired PS) or radiological progressive disease $(n=24)$, whereas one patient was on treatment at the time these results were reported. All patients received the planned dose on time, with the exception of three patients experiencing a 
Table I Demographics, baseline characteristics, and prior treatments in the triweekly, weekly, and sorafenib-resistant cohorts

\begin{tabular}{|c|c|c|c|c|c|c|}
\hline \multirow[b]{2}{*}{ Characteristics } & \multicolumn{2}{|c|}{ Triweekly } & \multicolumn{2}{|c|}{ Weekly } & \multicolumn{2}{|c|}{ Sorafenib resistant ${ }^{a}$} \\
\hline & $\mathbf{N}=\mathbf{2 7}$ & $\%$ & $N=13$ & $\%$ & $\mathbf{N}=12$ & $\%$ \\
\hline \multicolumn{7}{|l|}{ Sex } \\
\hline Male & 22 & 82 & 10 & 77 & 9 & 75 \\
\hline Female & 5 & 18 & 3 & 23 & 3 & 25 \\
\hline \multicolumn{7}{|c|}{ ECOG performance status } \\
\hline 0 & 19 & 70 & 7 & 54 & 6 & 50 \\
\hline 1 & 8 & 30 & 6 & 46 & 6 & 50 \\
\hline \multicolumn{7}{|l|}{ Child-Pugh class } \\
\hline A & 20 & 74 & 13 & 100 & II & 92 \\
\hline B & 7 & 26 & - & - & I & 8 \\
\hline \multicolumn{7}{|l|}{ BLCL staging } \\
\hline B & 5 & 18 & 2 & 18 & 1 & 8 \\
\hline C & 22 & 82 & 11 & 82 & 11 & 92 \\
\hline \multicolumn{7}{|l|}{ CLIP score } \\
\hline Median & 3 & - & 2 & - & 3 & - \\
\hline 0 & 2 & 7 & - & - & I & 8 \\
\hline I & 2 & 7 & I & 7 & 2 & 16 \\
\hline 2 & 9 & 33 & 6 & 46 & 3 & 25 \\
\hline 3 & 10 & 37 & 4 & 31 & 5 & 42 \\
\hline 4 & 4 & 15 & 2 & 15 & । & 8 \\
\hline \multicolumn{7}{|l|}{ Prior treatments } \\
\hline Transplantation & 1 & 3 & - & - & - & - \\
\hline Ablation & 2 & 7 & 2 & 15 & I & 8 \\
\hline Resection & 9 & 33 & 6 & 46 & 2 & 16 \\
\hline TACE & 16 & 59 & 9 & 69 & 7 & 58 \\
\hline Systemic therapy & 14 & 52 & 9 & 69 & 12 & 100 \\
\hline \multicolumn{7}{|c|}{$\alpha$-fetoprotein $\left(n g \mathrm{ml}^{-1}\right)$} \\
\hline Median & 749 & - & 209 & - & 31 & - \\
\hline Mean & 6409 & - & 14795 & - & 5434 & - \\
\hline Range & $2-47031$ & - & $2-170612$ & - & $2-47031$ & - \\
\hline \multicolumn{7}{|c|}{ Total bilirubin (mg dl-1) } \\
\hline Median & 1.1 & - & 1.2 & - & 1.0 & - \\
\hline Range & $0.4-3.8$ & - & $0.6-3.7$ & - & $0.7-2.8$ & - \\
\hline \multicolumn{7}{|l|}{ Tumour size $(\mathrm{cm})$} \\
\hline Median & 12.4 & - & 6.2 & - & 9.6 & - \\
\hline Range & $2.9-42.8$ & - & $2.3-14.0$ & - & $3.3-31.4$ & - \\
\hline \multicolumn{7}{|l|}{ Tumour morphology } \\
\hline Multinodular & 23 & 85 & 13 & 100 & 10 & 83 \\
\hline Uninodular & 2 & 7 & - & - & I & 8 \\
\hline No liver disease & 2 & 7 & - & - & I & 8 \\
\hline \multicolumn{7}{|c|}{ Macrovascular invasion and/or extrahepatic spread } \\
\hline Yes & 20 & 74 & 9 & 69 & 8 & 67 \\
\hline No & 7 & 26 & 4 & 31 & 4 & 33 \\
\hline
\end{tabular}

Abbreviations: $\mathrm{DFI}=$ disease-free interval; $E C O G=$ Eastern Cooperative Oncology Group: $\mathrm{BLCL}=$ Barcelona Clinic Liver Cancer; $\mathrm{CLIP}=$ Cancer of the Liver Italian Program; TACE $=$ transarterial chemoembolisation. Notes: Five patients (18\%, including four Child-Pugh class-B patients and one cirrhotic patient) in the triweekly cohort and one cirrhotic patient in the weekly cohort did not previously receive TACE or systemic therapies. Nine patients (33\%) in the triweekly cohort and six patients (46\%) in the weekly cohort had previously received both TACE and systemic therapies. Two patients (7\%) in the triweekly cohort and one patient (8\%) in the weekly cohort had previously received more than one prior systemic therapies. The DFI in the transplanted patient was 26.1 months, whereas the median DFI in patients who underwent resection $(n=15)$ or ablation $(n=4)$ were 17.7 months (range, 3.2-52.1) and 24.4 months (range, 3.1-47.4), respectively. There was no correlation between the duration of DFI and the overall survival time on this study treatment (Spearman's $r=0.34 ; P=0.14$ ). ${ }^{~}$ Including five and seven patients enrolled in the triweekly and weekly cohorts, respectively.
1-4 weeks delay in dosing as a result of their symptomatic deterioration.

Regardless of causality, most commonly experienced AEs were chills, pain, and asthenia (Table 2). Globally, only 41 (30\%) out of all registered AEs were considered related to study drug, and the most frequent were chills, noted over 27 cycles (27\%), and transient blood pressure increase, reported over six cycles (6\%). No grade 3-4 drug-related toxicity was observed. Among seven patients with Child-Pugh class-B status, five (71\%) experienced grade 1-2 drug-related AEs. These events were short lived and temporally related to study drug infusion, as they occurred approximately $30 \mathrm{~min}$ after the start of first infusions and lasted for about $20 \mathrm{~min}$.

A total of 101 weekly cycles (range, 5-12) were delivered in the weekly cohort. There was no exacerbation of toxicity profile by using the weekly schedule. Neither grade 3-4 drug-related toxicities nor toxicity-related deaths were reported. Most common drug-related toxicity was grade 1-2 chills experienced by 10 patients $(77 \%)$ over 30 infusions $(30 \%)$. Eleven patients discontinued treatment for radiological progression, one patient for Child-Pugh progression, and one for impaired PS.

\section{Efficacy}

Overall efficacy results for the triweekly, weekly, and sorafenibresistant cohorts are summarised in Table 3.

Following the first study stage $(n=16)$ in the triweekly cohort, five patients (31\%; 95\% CI: $11-59 \%)$ resulted in progression free at the 12 -week time point. Even though this proportion was lower than the protocol-specified minimum requirement, the accrual was restarted and also took into account the safety/tolerability profile of the experimental drug. After the second study stage $(n=27)$, eight patients (30\%; 95\% CI: $14-50 \%)$ were progression free at 12 weeks and the median PFS was 2.3 months (95\% CI: $1.7-2.9$ months).

By radiological tumour assessment, the DCR was $30 \%$. A complete response (4\%), currently ongoing after 20 months of therapy, was observed in a 76-year-old Child-Pugh class-B patient, who had been previously refractory to sorafenib after 2 months of therapy (Figure 1). A partial response (4\%), with an overall $78 \%$ tumour reduction, was reported in a 65 -year-old Child-Pugh classB patient (Figure 2). In addition, tumour shrinkages were detected in four out of six patients (22\%) who experienced stable disease as best response (Figure 3). This subset of eight patients who achieved disease control received a median of five treatment cycles (range, 4-25) and had a median PFS of 4.3 months (range, 3 to $20+$ months).

At a median follow-up of 26.1 months, the median survival time was 8.9 months (95\% CI: 7.5-10.2 months), as shown in Figure 4. The estimated survival rates at 12 and 24 months were 26 and $18 \%$, respectively. Five patients $(18 \%)$ received subsequent anticancer therapy (all patients were treated with sorafenib).

In post hoc subset analyses, there were no differences in survival for patients either untreated (median, 8.6 months) or previously treated with systemic therapy (median, 9.5 months). The subset of Child-Pugh class-B patients $(n=7)$ had 6 -month PFS and OS rates of 17 and $29 \%$, respectively.

In an univariate Cox proportional hazards model fitted by using the baseline characteristic variables listed in Table 1, patient's PS of 0 and CLIP scores of $0-3$ were the only factors significantly associated with improved survival $(P=0.011$ and 0.048 , respectively).

Paired serum samples (pre- and post-first treatment cycle) for chromogranin A ( $\mathrm{CgA})$ assessments were available from 18 patients. At baseline, serum CgA levels (median, $161 \mathrm{ng} \mathrm{ml}^{-1}$; 95\% CI: 123-303) did not correlate with either PFS (Spearman's $r=0.10 ; P=0.60)$ or OS $(r=0.007 ; P=0.97)$. After the first treatment cycle, CgA levels decreased (median, $121 \mathrm{ng} \mathrm{ml}^{-1} ; 95 \%$ CI: 65-212), even though the difference did not reach statistical 
Table 2 Worst grade per patient of adverse events, irrespective of relationship to study drug, reported in the triweekly cohort

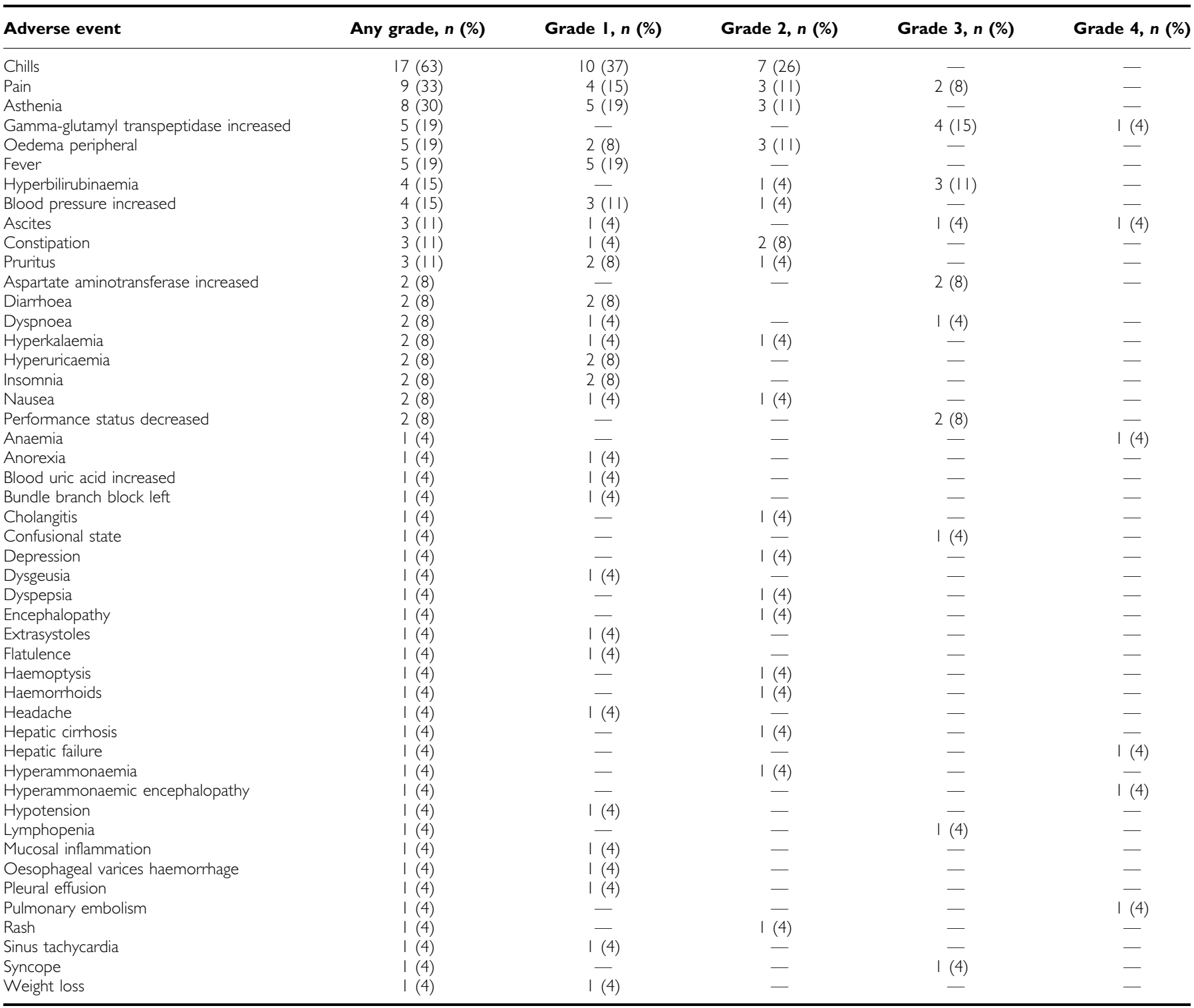

Table 3 Overall efficacy results in the triweekly, weekly, and sorafenib-resistant cohorts

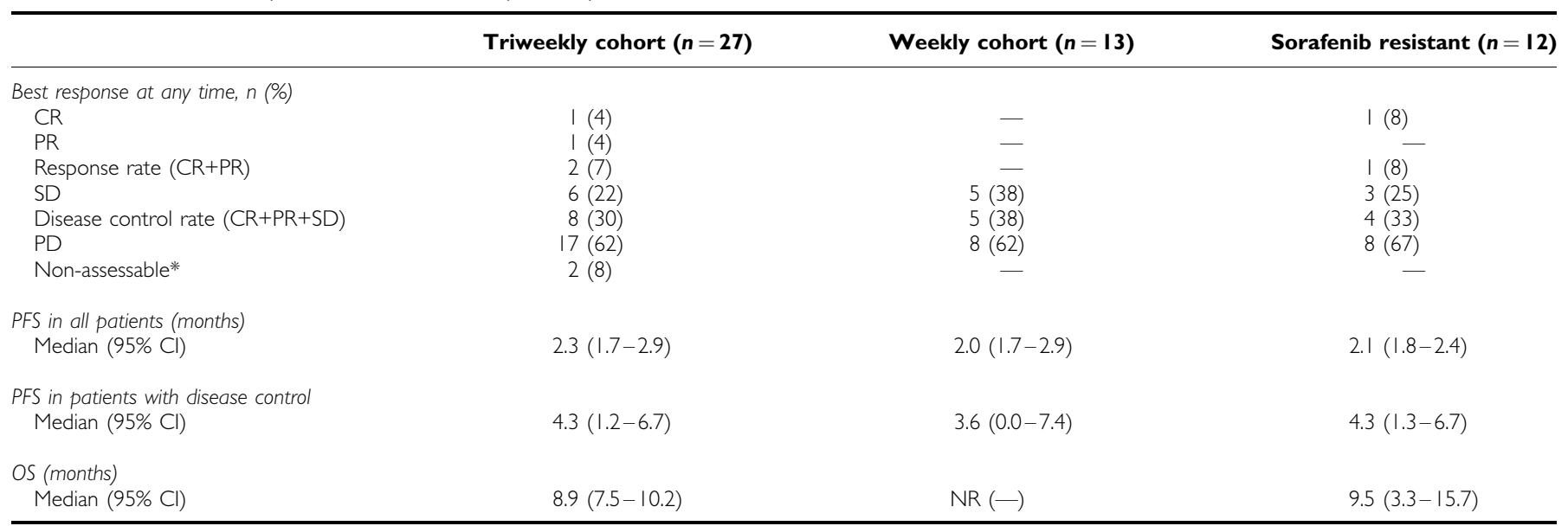

Abbreviations: $\mathrm{Cl}=$ confidence interval; $C R=$ complete response; $N R=$ not reached; $O S=$ overall survival; $P D=$ progressive disease; $P F S=$ progression-free survival; $\mathrm{PR}=$ partial response; $\mathrm{SD}=$ stable disease. *Two patients were withdrawn from the study before their first restaging because of symptonatic deterioration. 

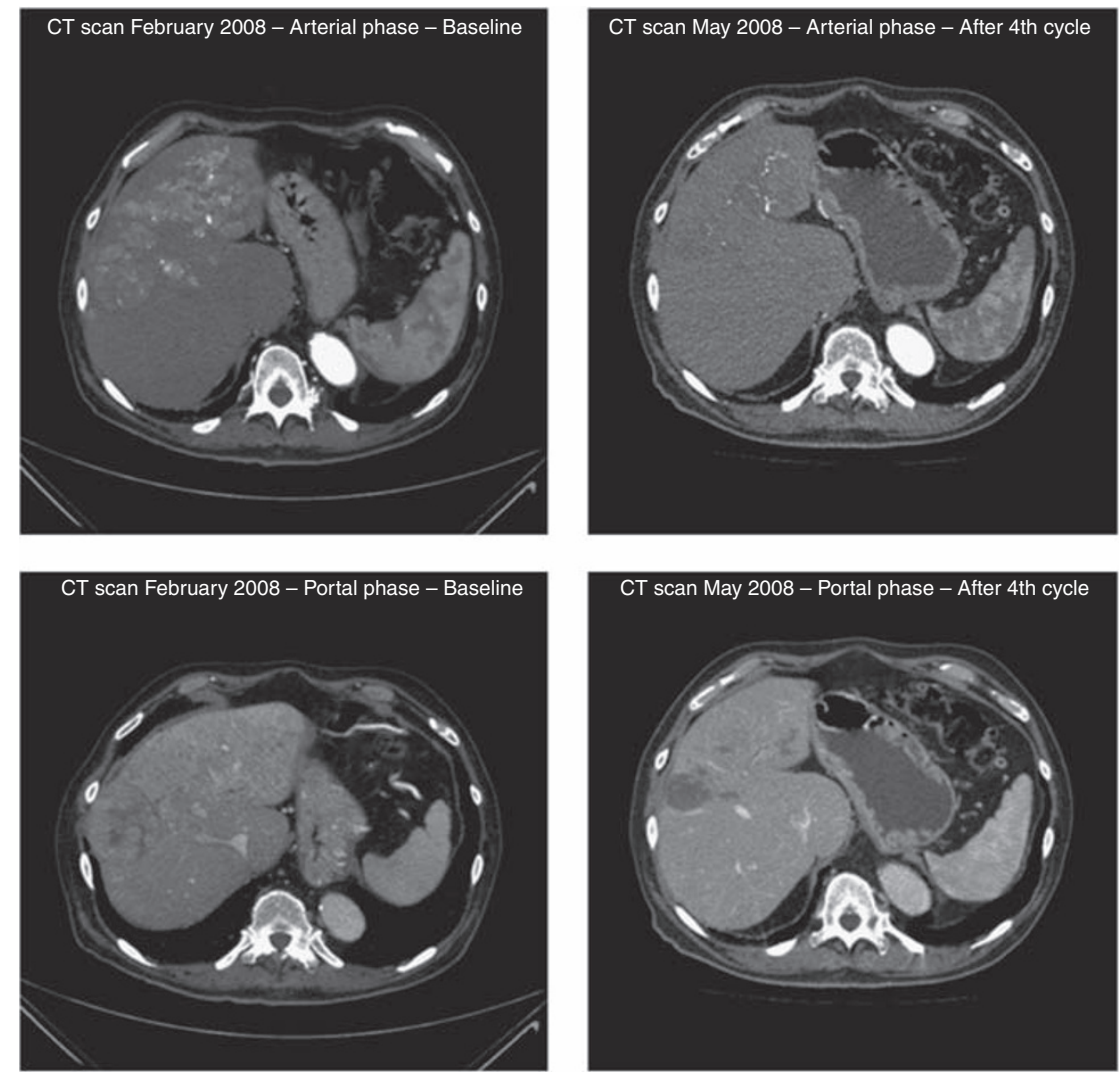

Figure I Baseline and post-treatment computed tomography scans in a 76-year-old sorafenib-refractory male patient presenting with a large $\left(6.1 \times 6.1 \mathrm{~cm}^{2}\right)$ hepatic mass and extensive multifocal lesions (scans on the left), who had a complete necrosis of the primary lesion and absence of any foci of contrast-enhancement in the remaining parenchyma (scans on the right).
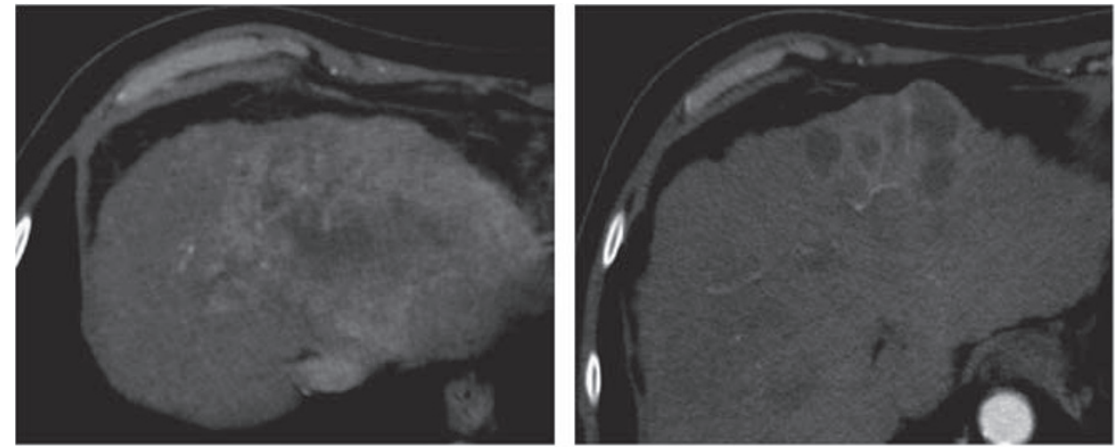

Figure 2 Baseline and post-treatment computed tomography scans in a 65-year-old Child-Pugh class-B male patient presenting with a large $\left(10.6 \times 8.0 \mathrm{~cm}^{2}\right)$ hepatic mass (scan on the left), who experienced tumour shrinkage of the primary tumour after the fourth cycle of treatment (scan on the right).

significance $(P=0.24$, Wilcoxon test), and at this time point tended to correlate inversely with survival $(r=-0.44 ; P=0.06)$. Median survival in patients with post-first cycle CgA levels lower and higher than the median value were 12 and 8.6 months, respectively.

In the weekly cohort, five patients $(38 \%)$ had stable disease for a median duration of 3.6 months. After a median follow-up time of 9.0 months (95\% CI: 4.7-13.3 months), the median survival has not yet been reached. Overall, there were no apparent differences in either tolerability or activity between the two dosing schedules, with the triweekly administration being more preferable than weekly for patient's convenience.
Overall, the subset of 12 sorafenib-pretreated patients reported a response rate of $8 \%$ and a DCR of $33 \%$, whereas the median PFS and survival were 2.1 and 9.5 months, respectively (Figure 5). All patients discontinued study treatment because of radiologically documented progressive disease. Median survival was 13 months, if recorded from the date of sorafenib therapy initiation. The median ratio between the PFS durations on the previous sorafenib therapy (median, 2.3 months; 95\% CI: $2.1-2.4$ ) and on the current study therapy (median, 2.1 months; 95\% CI: $1.8-2.4$ ) was 0.72 , with four patients $(33 \%)$ experiencing PFS durations equal or longer than those experienced while on the previous sorafenib therapy. 


\section{2}

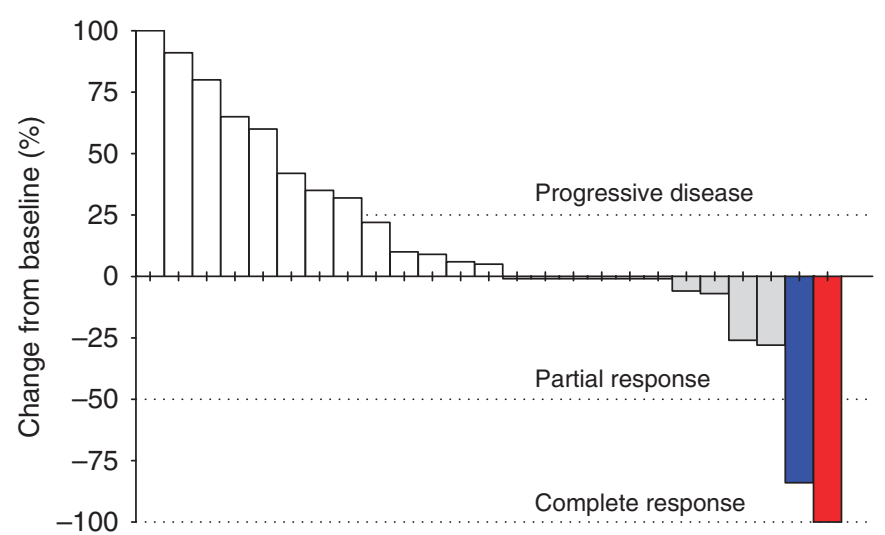

Figure 3 Waterfall plot of changes in tumour size in the overall population of evaluable patients $(n=25)$ treated with the triweekly schedule. Data are largest response during study, irrespective of the time of assessment. Notes: Patients with complete response $(n=1)$ or partial response $(n=1)$ according to the $\mathrm{WHO}$ criteria are shown in red and blue bars, respectively, while those with stable disease $(S D, n=6)$ or progressive disease $(P D, n=17)$ are shown in grey and white bars, respectively. Four patients with SD had tumour shrinkage, whereas two patients with SD did not. Nine patients had PD in target lesions, while eight patients had PD in non-target lesions.

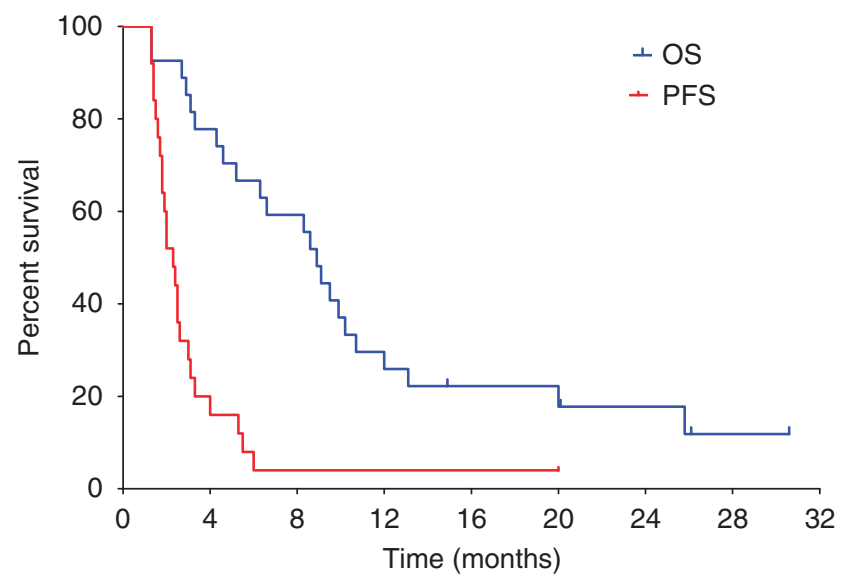

Figure 4 Kaplan-Meier curves of progression-free (red line) and overall survival (blue line) in patients treated with the triweekly schedule $(n=27)$ Vertical ticks denote censored observations.

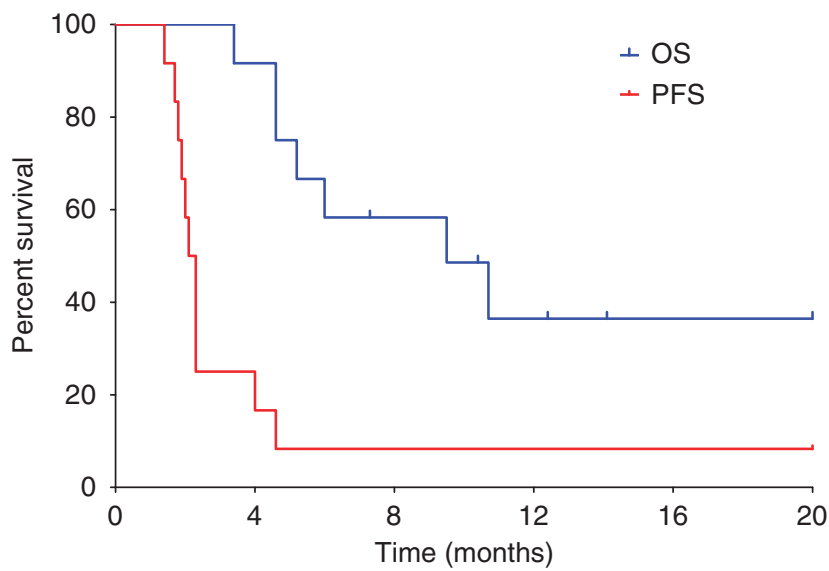

Figure 5 Kaplan-Meier curves of progression-free (red line) and overall survival (blue line) in the cohort of 12 sorafenib-resistant patients, enrolled in the triweekly schedule $(n=5)$ or weekly schedule $(n=7)$. Vertical ticks denote censored observations.

\section{DISCUSSION}

The patients enrolled in this study had a poor-prognosis disease as the majority presented with BCLC C stage. The natural history of these patients, if untreated, results in the median survival of about 6 months (Llovet et al, 2008a). Specifically, median survival times were 4.2 months (Cheng et al, 2009) and 7.9 months (Llovet et al, 2008b) in Asian and European patient populations, respectively, who received placebo only in two randomised phase III trials testing sorafenib as first-line treatment of HCC.

Most patients (52\%) had previously received one or more systemic therapies and $26 \%$ did not present with a preserved liver function (Child-Pugh class-B). Our findings indicated that treatment with NGR-hTNF was very well tolerated. There was no drug-related grade 3-4 toxicity, most common grade 1-2 AEs being transient constitutional symptoms experienced by half of patients during first infusions.

NGR-hTNF induced a confirmed response rate of 7\%, a DCR of $30 \%$, and a median survival of 8.9 months. The 3-month PFS rate was lower than the protocol-prespecified target deemed worthy of further testing. However, about one-third of patients remained progression free for a median time of more than 4 months. Of note, similar results were also observed in a subset of 12 patients resistant/refractory to sorafenib, with a confirmed response rate of $8 \%$, a DCR of $33 \%$, and a median survival time of 9.5 months. There was also evidence of the antitumour activity of NGR-hTNF in patients either untreated or previously treated with systemic therapy. Indeed, a sorafenib-refractory patient had a durable complete response lasting more than 20 months and an untreated Child-Pugh class-B patient had a partial response. These tumour regressions in highly vascularised tumours might establish a clinical proof-of-principle of the antivascular mode of action of NGR-hTNF, previously documented by DCE-MRI evaluation (Gregorc et al, 2010; van Laarhoven et al, 2010). Even though dynamic imaging assessment was not performed in this study, it is interesting to note the decrease, although not statistically significant, of serum $\mathrm{CgA}$ levels registered after the first cycle. Indeed, increased CgA levels, in concert with high circulating TNFreceptor levels, have been reported as a protective factor against TNF-induced vascular damage (Ferrero et al, 2004), as well as an independent poor-prognosis indicator (Gregorc et al, 2007).

Historically, the design of clinical trials in HCC has been highly challenging because of the underlying liver dysfunction. For testing a new drug in a phase II trial, a randomised design has been recommended as more appropriate than single-armed trial, a timerelated end point more critical than response rate, and the time to radiological progression (TTP) more adequate than PFS, owing to the latter representing a vulnerable composite end point in HCC because death from cirrhosis might confound detection of treatment effect (Llovet et al, 2008a). Therefore, the results of this uncontrolled phase II study might only be evaluated in the context of other trial results obtained in similar advanced-stage HCC patients. Although comparison with historical controls is always flawed and particularly challenging in HCC patients because of differences in either disease stage or previous treatments, the overall outcomes reported in this study seem to be in the range of those reported in phase II trials testing molecular therapies as single agents. Indeed, targeted monotherapies, mainly tested in untreated HCC patients, were associated to response rate of 0-13\%, median PFS of 1.4-6.9 months, and median survival of 6.2-13 months (Table 4), underlining the issue of patient selection bias for uncontrolled phase II trials. The largest trials in patients with advanced-stage HCC are the SHARP (sorafenib HCC assessment randomised protocol) (Llovet et al, 2008b) and the Asia-Pacific liver (Cheng et al, 2009) phase III studies evaluating sorafenib vs placebo. Both studies enrolled previously untreated patients. Median survival of 10.7 months and TTP of 5.5 months were reported for sorafenib in the SHARP trial, and median 
Table 4 Summary of efficacy results reported in recent phase II trials using molecularly targeted therapies and in phase III trials using sorafenib for the treatment of $\mathrm{HCC}$

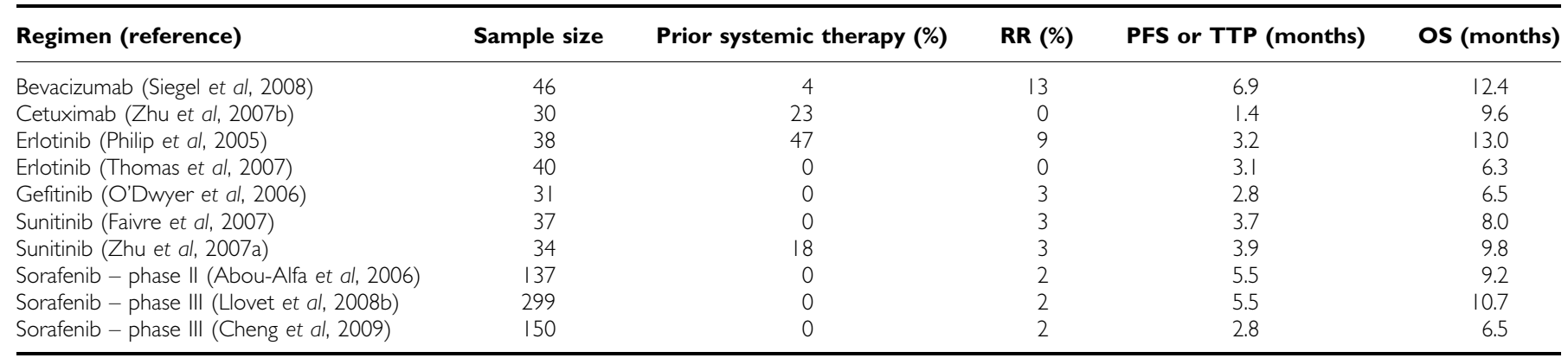

Abbreviations: $\mathrm{HCC}=$ hepatocellular carcinoma; $\mathrm{PFS}=$ progression-free survival; $\mathrm{OS}=$ overall survival; $\mathrm{RR}=$ response rate; $\mathrm{TTP}=$ time to radiological progression

survival of 6.5 months and TTP of 2.8 months were reported for sorafenib in the Asia-Pacific study. The objective response rate was however around $3 \%$ in both studies.

In conclusion, the evidence of preliminary antitumour activity noted with NGR-hTNF in this study seems to deserve further testing in advanced HCC. The mode of action, along with a favourable tolerability profile characterised by the lack of drugrelated grade 3-4 toxicity, could also facilitate the incorporation of NGR-hTNF into combination regimens for HCC patients. Consistently, in preclinical models even low concentrations of NGR-TNF were able to increase the activity of a variety of chemotherapeutic agents (Curnis et al, 2002b; Sacchi et al, 2006). These synergistic effects were deemed owing to the tumour capillary network damage, thereby increasing the chemotherapy uptake. Moreover, a safe clinical toxicity profile was reported combining NGR-hTNF with a chemotherapeutic agent used in HCC such as doxorubicin (Gregorc et al, 2009). In addition, there is a strong mechanistic rationale for an enhanced activity of vascular-damaging agents when combined with antiangiogenic drugs (Shaked et al, 2006).

Finally, given the drawbacks inherent in interpreting results from single-arm trial in a heterogeneous malignancy such as HCC, a more suitable approach for further evaluation might be a randomised study design by using time-to-event as the primary end point. The potential clinical benefit of NGR-hTNF, therefore, needs to be prospectively assessed against the best investigator's option in sorafenib-pretreated patients with advancedstage HCC.

\section{REFERENCES}

Abou-Alfa GK, Schwartz L, Ricci S, Amadori D, Santoro A, Figer A, De Greve J, Douillard JY, Lathia C, Schwartz B, Taylor J, Moscovici M, Saltz LB (2006) Phase II study of sorafenib in patients with advanced hepatocellular carcinoma. J Clin Oncol 24: 4293

Arap W, Pasqualini R, Ruoslahti E (1998) Cancer treatment by targeted drug delivery to tumor vasculature in a mouse model. Science 279: $377-380$

Carswell EA, Old LJ, Kassel RL, Green S, Fiore N, Williamson B (1975) An endotoxin-induced serum factor that causes necrosis of tumours. Proc Natl Acad Sci 72: 3666-3670

Cheng AL, Kang YK, Chen Z, et Tsao CJ, Qin S, Kim JS, Kim JS, Luo R, Feng J, Ye S, Yang TS, Xu J, Sun Y, Liang H, Liu J, Wang J, Tak WJ, Pan H, Burok K, Zou J, Voliotis D, Guan Z (2009) Efficacy and safety of sorafenib in patients in the Asia-Pacific region with advanced hepatocellular carcinoma: a phase III randomised, double-blind, placebo-controlled trial. Lancet Oncol 10: $25-34$

Corti A, Ponzoni M (2004) Tumor vascular targeting with tumor necrosis factor alpha and chemotherapeutic drugs. Ann NY Acad Sci 1028: $104-112$

Curnis F, Arrigoni G, Sacchi A, Fischetti L, Arap W, Pasqualini R, Corti A (2002a) Differential binding of drugs containing the NGR motif to CD13 isoforms in tumor vessels, epithelia, and myeloid cells. Cancer Res 62: $867-874$

Curnis F, Sacchi A, Borgna L, Magni F, Gasparri A, Corti A (2000) Enhancement of tumor necrosis factor alpha antitumor immunotherapeutic properties by targeted delivery to aminopeptidase N (CD13). Nat Biotechnol 18: 1185-1190

Curnis F, Sacchi A, Corti A (2002b) Improving chemotherapeutic drug penetration in tumors by vascular targeting and barrier alteration. $J$ Clin Invest 110: $475-482$

Faivre SJ, Raymond E, Douillard J, Boucher E, Lim HJ, Kim JS, Lanzalone S, Lechuga MJ, Sherman L, Cheng A (2007) Assessment of safety and drug-induced tumor necrosis with sunitinib in patients with unresectable hepatocellular carcinoma (HCC). J Clin Oncol 25: 3546
Ferrero E, Scabini S, Magni E, Foglieni C, Belloni D, Colombo B, Curnis F, Villa A, Ferrero ME, Corti A (2004) Chromogranin A protects vessels against tumor necrosis factor alpha-induced vascular leakage. FASEB $J$ 20: 20

Gregorc V, Citterio C, Vitali G, Spreafico A, Scifo P, Borri A, Donadoni G, Rossoni G, Corti C, Caligaris-Cappio F, Del Maschio A, Esposito A, De Cobelli F, Dell'Acqua F, Troysi A, Bruzzi P, Lambiase A, Bordignon C (2010) Defining the optimal biological dose of NGR-hTNF, a selective vascular targeting agent, in advanced solid tumours. Eur J Cancer 46: $198-206$

Gregorc V, Santoro A, Bennicelli E, Punt CJA, Citterio G, Timmer-Bonte JNH, Caligaris-Cappio F, Lambiase A, Bordignon C, van Herpen CML (2009) Phase Ib study of NGR-hTNF, a selective vascular targeting agent, administered at low doses in combination with doxorubicin to patients with advanced solid tumours. Br J Cancer 101: 219-224

Gregorc V, Spreafico A, Floriani I, Colombo B, Ludovini V, Pistola L, Bellezza G, Viganò MG, Villa E, Corti A (2007) Prognostic value of circulating chromogranin A and soluble tumor necrosis factor receptors in advanced nonsmall cell lung cancer. Cancer 110: 845-853

Greten TF, Korangy F, Manns MP, Malek NP (2009) Molecular therapy for the treatment of hepatocellular carcinoma. $\mathrm{Br}$ J Cancer 100: 19-23

Lejeune FJ, Lienard D, Matter M, Ruegg C (2006) Efficiency of recombinant human TNF in human cancer therapy. Cancer immun 6: 6-23

Llovet JM, Di Bisceglie AM, Bruix J, Kramer BS, Lencioni R, Zhu AX, Sherman M, Schwartz M, Lotze M, Talwalkar J, Gores GJ (2008a) Design and endpoints of clinical trials in hepatocellular carcinoma. J Natl Cancer Inst 100: 698-711

Llovet JM, Ricci S, Mazzaferro V, Hilgard P, Gane E, Blanc JF, Cosme de Oliveira A, Santoro A, Raoul JL, Forner A, Schwartz M, Porta C, Zeuzem S, Bolondi L, Greten TF, Galle PR, Seitz JF, Borbath I, Häussinger D, Giannaris T, Shan M, Moscovici M, Voliotis D, Bruix J (2008b) Sorafenib in advanced hepatocellular carcinoma. N Engl J Med 359: 378-390

O'Dwyer PJ, Giantonio BJ, Levy DE, Kauh JS, Fitzgerald DB, Benson AB (2006) Gefitinib in advanced unresectable hepatocellular carcinoma: 
results from the Eastern Cooperative Oncology Group's Study E1203.

Philip PA, Mahoney MR, Allmer C, Thomas J, Pitot HC, Kim G, Donehower RC, Fitch T, Picus J, Erlichman C (2005) Phase II study of erlotinib (OSI-774) in patients with advanced hepatocellular cancer. J Clin Oncol 23: $6657-6663$

Rangel R, Sun Y, Guzman-Rojas L, Ozawa MG, Sun J, Giordano RJ, Van Pelt CS, Tinkey PT, Behringer RR, Sidman RL, Arap W, Pasqualini R (2007) Impaired angiogenesis in aminopeptidase N-null mice. Proc Natl Acad Sci USA 104: 4588-4593

Sacchi A, Gasparri A, Gallo-Stampino C, Toma S, Curnis F, Corti A (2006) Synergistic antitumor activity of cisplatin, paclitaxel, and gemcitabine with tumor vasculature-targeted tumor necrosis factor-alpha. Clin Cancer Res 12: 175-182

Shaked Y, Ciarrocchi A, Franco M, Lee CR, Man S, Cheung AM, Hicklin DJ, Chaplin D, Stuart Foster F, Benezra R, Kerbel RS (2006) Therapy-induced acute recruitment of circulating endothelial progenitor cells to tumors. Science 22: $1785-1787$

Siegel AB, Cohen EI, Ocean A, Lehrer D, Goldenberg A, Knox JJ, Chen H, Clark-Garvey S, Weinberg A, Mandeli J, Christos P, Mazumdar M, Popa E, Brown Jr RS, Rafii S, Schwartz JD (2008) Phase II trial evaluating the clinical and biologic effects of bevacizumab in unresectable hepatocellular carcinoma. J Clin Oncol 26: 2992-2998 J Clin Oncol 24: 4143

Thomas MB, Chadha R, Glover K, Wang X, Morris J, Brown T, Rashid A, Dancey J, Abbruzzese JL (2007) Phase 2 study of erlotinib in patients with unresectable hepatocellular carcinoma. Cancer 110: 1059-1067

van Laarhoven HWM, Fiedler W, Desar IME, van Asten JJA, Sandrine Marréaud S, Lacombe D, Govaerts AS, Bogaerts J, Lasch P, TimmerBonte JNH, Lambiase A, Bordignon C, Punt CJA, Heerschap A, van Herpen CML (2010) Phase I clinical and magnetic resonance imaging study of the vascular agent NGR-hTNF in patients with advanced cancers (European Organization for Research and Treatment of Cancer Study 16041). Clin Cancer Res 16: 1315-1323

Zhu AX, Sahani DV, di Tomaso E, Duda D, Sindhwani V, Yoon SS, Blaszkowsky LS, Clark JW, Ryan DP, Jain RK (2007a) A phase II study of sunitinib in patients with advanced hepatocellular carcinoma. J Clin Oncol 25: 4637

Zhu AX, Stuart K, Blaszkowsky LS, Muzikansky A, Reitberg DP, Clark JW, Enzinger PC, Bhargava P, Meyerhardt JA, Horgan K, Fuchs CS, Ryan DP (2007b) Phase 2 study of cetuximab in patients with advanced hepatocellular carcinoma. Cancer 110: $581-589$

This work is licensed under the Creative
(C) (2) This work is licensed under the Creative
BY NC SA Commons Attribution-NonCommercial-Share Alike 3.0 Unported License. To view a copy of this license, visit http://creativecommons.org/licenses/by-nc-sa/3.0/ 